\title{
OPTICAL, HST, AND ROSAT OBSERVATIONS OF BAL QSOS
}

\author{
MICHAEL KOPKO, JR., DAVID A. TURNSHEK, and AND BRIAN R. ESPEY \\ Department of Physics and Astronomy, University of Pittsburgh
}

We consider multiwavelength observations of Broad Absorption Line (BAL) QSOs which include optical spectrophotometry supplemented by HST-FOS UV spectrophotometry and/or ROSAT-PSPC x-ray observations. For moderate to high redshift objects, increasing the wavelength coverage into the UV permits observation of BALs due to different ions of the same element for several different elements for the first time. By employing appropriate assumptions, ionic column densities as a function of outflow velocity can be derived from these observations, resulting in constraints on the level of ionization and chemical composition of the BAL region (BALR) gas and the photoionization model itself.

Under the assumption that the BALR gas is photoionized, analysis with the photoionization code CLOUDY developed by Ferland suggests that BALR abundances must be generally enhanced at least 10 to 100 times solar values. The wide range in ionization level observed in some BAL QSOs appears to indicate that for a given outflow velocity a range of ionization parameters applies.

HST FOS UV observations of the low-redshift weak-BAL QSO PG0043+039 show a deficit of flux shortward of $\sim 2200 \AA$ which is consistent with intrinsic dust-extinction by $\mathrm{SMC}$-like dust with $\mathrm{E}(\mathrm{B}-\mathrm{V}) \simeq 0.1$. There is also evidence for significant amounts of FeII emission and a lack of narrow-lined [OIII] emission. The details of this result are reported in a publication by the HST Quasar Absorption Line Key Project Team (Turnshek et al.).

From observations of BAL QSO emission-line profiles, the absence of significant emission components expected from resonance line scattering has generally been used to infer that BALR covering factors are $<0.2$. However, given the likelihood that narrow-line [OIII] emission is emitted isotropically, the high incidence of BALs in samples of QSOs with weak-[OIII] emission and strong-FeII emission suggests that the BALR covering factor may be large in some subset of QSOs. The presence of dust in the BAL region could provide a mechanism to destroy photons which were resonance line scattered in the BAL region.

Pointed ROSAT PSPC observations of a sample of 5 moderate-redshift $(\mathrm{z} \simeq$ 2) BAL QSOs show that $1.9<\alpha_{O X}<2.5\left(\mathrm{f}_{\nu} \sim \nu^{-\alpha}\right)$ is consistent with the observations. This is well outside the range of $1.3-1.8$ quoted in the literature for moderate-redshift radio quiet QSOs. This indicates that either significant photoelectric absorption occurs in the BALR or that there is decreased $\mathbf{x}$-ray production along the direction of BALR outflow. The presence of photoelectric absorption would be consistent with enhanced BALR elemental abundances.

T. J.-L. Courvoisier and A. Blecha: Multi-Wavelength Continuum Emission of AGN, 450.

(C) 1994 IAU. Printed in the Netherlands. 